Cite this: Food Funct., 2014, 5, 894

\title{
Grain sorghum muffin reduces glucose and insulin responses in men
}

\author{
Nicole M. Poquette, Xuan Gu and Sun-Ok Lee*
}

Diabetes and obesity have sparked interest in identifying healthy, dietary carbohydrates as functional ingredients for controlling blood glucose and insulin levels. Grain sorghum has been known to be a slowly digestible cereal; however, research is limited on its health effects in humans. The objectives of this study were to measure the contents of functional starch fractions, SDS (slowly-digestible starch) and RS (resistant starch), and to investigate the effects of grain sorghum on postprandial plasma glucose and insulin levels in 10 healthy men. A whole-wheat flour muffin (control) was compared with the grain sorghum muffin with both muffins containing $50 \mathrm{~g}$ of total starch. Using a randomized-crossover design, male subjects consumed treatments within a one-week washout period, and glucose and insulin levels were observed at 15 minutes before and $0,15,30,45,60,75,90,120,180$ minutes after consumption. The mean glucose responses reduced after consuming grain sorghum, particularly at 45-120 minute intervals, and mean insulin responses reduced at 15-90 minute intervals compared to control $(P<0.05)$. The mean incremental area under the curve (iAUC) was significantly lowered for plasma glucose responses about an average of $35 \%$ from $3863 \pm 443$ to $2871 \pm 163 \mathrm{mg}(\sim 3 \mathrm{~h}) \mathrm{dL}^{-1}(P<0.05)$. Insulin responses also reduced significantly from $3029 \pm 965 \mu \mathrm{U}(\sim 3 \mathrm{~h}) \mathrm{L}^{-1}$ for wheat to $1357 \pm 204$ with sorghum $(P<0.05)$. Results suggest that grain sorghum is a good functional ingredient to assist in managing glucose and insulin levels in healthy individuals.

Received 20th September 2013 Accepted 27th January 2014

DOI: $10.1039 / c 3 f 060432 b$

www.rsc.org/foodfunction sorghum is mainly used as animal feed in Australia and the U.S. ${ }^{5}$ However, demand for finding alternative and functional ingredients has prompted expanded use in markets. Similar to other cereal grains, sorghum is mostly composed of starch including the pericarp of the grain, and the starch content may range from $55-76 \%$ depending upon crop and cultivar..$^{5-9}$ Grain sorghum is comparable to maize in terms of composition and the starch structure, ${ }^{5,8-11}$ however, its protein properties distinguish the grain from other cereals. ${ }^{5-9}$ The protein matrix has been identified as being one of the key components to its indigestibility; however, processing methods have also shown to increase digestibility. ${ }^{8-10}$ Research has identified grain sorghum as a potential functional ingredient in food applications, ${ }^{8,12}$ however, researchers have also reported conflicting results based on sorghum sources and processing conditions. ${ }^{7}$ Despite conflicting results, studies using whole grain sorghum have shown strong evidence of reduced plasma glucose levels after consumption in both human and animal models. ${ }^{6,12-15}$

When analyzing grain sorghum, the health benefits associated with consumption have been attributed to the indigestible properties due to its structure and composition. Dietary fiber and tannin content in grain sorghum have been highlighted for nutritional potential, ${ }^{6,8}$ and additionally, sorghum is also an excellent alternative for gluten-free applications. With increasing demand of both healthier and allergen-free products in the market place, grain sorghum has a great potential for 
incorporation in numerous food applications as a healthy, dietary carbohydrate and could also potentially assist in the control of glucose and insulin levels in humans. The objective of this study was to investigate the efficacy of grain sorghum flour as a functional food ingredient by measuring the contents of functional starch fractions and the effects on postprandial plasma glucose and insulin responses in healthy humans.

\section{Materials and methods}

\section{Materials}

Whole grain sorghum flours from Bob's Red Mill (Milwaukie, OR, U.S.A.) and Archer Daniels Midland (Plainview, TX, U.S.A.) and whole wheat flour (Gold Medal, Minneapolis, MN, U.S.A.) were analyzed for starch fractions. All solvents and chemicals for the experiment were purchased from VWR international, Inc. (Suwanee, GA, U.S.A) or Sigma Chemical Co. (St. Louis, Mo., U.S.A). A kit for total starch determination was purchased from Megazyme International Ireland Ltd. (Bray Business Park, Wicklow, Ireland). A kit for plasma insulin determination was purchased from Mercodia (Uppsala, Sweden).

\section{Participant profile and study design}

A human study was approved by IRB at University of Arkansas and participants were recruited from University of Arkansas (Fayetteville, AR, U.S.A.). Written informed consent was obtained for all study participants. The study used a randomized-crossover design to investigate glucose and insulin responses. For the study, 10 healthy male subjects were selected after a screening session prior to the experiment to confirm fasting blood glucose levels $<100 \mathrm{mg} \mathrm{dL}^{-1}$ in addition to not diagnosed with any disease or illness and not taking any medication within the last 6 months. All subjects were nonsmokers and did not frequently consume more than two alcohol servings per week. The mean age, BMI and fasting blood glucose levels of the participant group were $25.1 \pm 4$ years, $24.2 \pm 2.8 \mathrm{~kg}$ $\mathrm{m}^{-2}$, and $92.2 \pm 6.4 \mathrm{mg} \mathrm{dL}{ }^{-1}$, respectively. Nine participants identified themselves as Asian or Asian American and one participant identified himself as Caucasian. All participants consented to experiment protocols and attended two Saturdays with a one-week washout period. Each subject consumed treatments after 10 hour fasting.

\section{Muffin preparation}

After the analyses of grain sorghum flours, the flour high in SDS and RS contents was used for the muffin product. For each experiment, two muffins which contained a total serving of $50 \mathrm{~g}$ of total starch were used. All raw materials were weighed separately for each treatment as shown in Table 1. Differences in water amounts were used to accommodate different amounts of flour. Dry ingredients were mixed first followed by wet ingredients, and then both were combined and mixed together. The batter was then weighed into individually greased-muffin liners to weigh approximate product samples. Muffins were baked at $425{ }^{\circ} \mathrm{F}$ for 15 minutes. Muffins were cooled for 10 minutes and stored until the following morning of experiment.
Table 1 Muffin ingredients

\begin{tabular}{lcc}
\hline Ingredient & Wheat muffin $(\mathrm{g})$ & Sorghum muffin $(\mathrm{g})$ \\
\hline Flour & 77.5 & 66.8 \\
Water & 46.3 & 37.7 \\
Egg & 28.8 & 28.8 \\
Butter & 20.0 & 20.0 \\
Vegetable oil & 7.5 & 7.5 \\
Sucralose & 3.0 & 3.0 \\
Baking soda & 1.8 & 1.8 \\
Salt & 1.3 & 1.3 \\
Vanilla extract & 1.8 & 1.8
\end{tabular}

Batter preparation and baking were conducted consistently each afternoon prior to experiment days. Analysis of total starch (TS) and starch fractions for the final muffin product was also carried out on each experiment day. Each subject consumed two muffins per serving for each treatment on respective experiment days. All ingredients were purchased at a local grocery store.

\section{Total starch content determination}

The total starch content of raw material flour samples and finished muffin products was determined following the manual (KOH format). A sample size of $100 \mathrm{mg}$ was dissolved in $2 \mathrm{M}$ $\mathrm{KOH}$ in a test tube and neutralized with sodium acetate buffer (1.2 M, pH 3.8) followed by $100 \mu \mathrm{L}$ of thermostable $\alpha$-amylase and $100 \mu \mathrm{L}$ of amyloglucosidase were immediately added. After the incubation period, samples were then diluted to $100 \mathrm{~mL}$ and $100 \mu \mathrm{L}$ of the homogenized sample was added to $3 \mathrm{~mL}$ of the glucose determination reagent (GOPOD) and incubated for 20 minutes at $50{ }^{\circ} \mathrm{C}$. After final incubation, the absorbance was immediately read at $510 \mathrm{~nm}$ and the content was determined according to the formula included in the manual.

\section{Starch fractions determination}

Starch fractions (RDS, SDS, and RS) were determined with the Englyst method $^{3}$ at the same time when subjects consumed muffins. Enzyme solution was prepared by dispersing $450 \mathrm{mg}$ of pancreatin in $20 \mathrm{~mL}$ of deionized water with stirring for 10 minutes, and after centrifugation $54 \mathrm{~mL}$ of the supernatant was mixed with $6 \mathrm{~mL}$ of amyloglucosidase (140 unit/mL). Muffin samples were ground and weighed in $50 \mathrm{~mL}$ centrifuge tubes on the basis of starch content, which were calculated to be the same as $800 \mathrm{mg}$ of flour, and $20 \mathrm{~mL}$ of sodium acetate buffer (0.5 M, pH 5.2) was added to the tubes and mixed well. After adding with $5 \mathrm{~mL}$ prepared enzyme in each tube, all the tubes were incubated horizontally in a water bath at $37{ }^{\circ} \mathrm{C}$ and 160 strokes per min. At $20 \mathrm{~min}$, an aliquot of $0.5 \mathrm{~mL}$ was pipetted from the tube and mixed with $20 \mathrm{~mL}$ of $80 \%$ ethanol for glucose determination (G20). Samples were replaced in the water bath in $30 \mathrm{~s}$. At $120 \mathrm{~min}$, another aliquot of $0.5 \mathrm{~mL}$ was pipetted from the tube to $20 \mathrm{~mL}$ of $80 \%$ ethanol and mixed well for glucose determination (G120). Glucose standard (20 mL of $25 \mathrm{mg} \mathrm{mL}^{-1}$ D-glucose in sodium acetate buffer introduced above) and buffer blank (20 mL of sodium acetate buffer) were carried out. A 
supernatant of $0.1 \mathrm{~mL}$ was pipetted into $3 \mathrm{~mL}$ of the GOPOD reagent and incubated at $50{ }^{\circ} \mathrm{C}$ for 20 minutes. The absorbance was read at $510 \mathrm{~nm}$. The starch fractions were calculated as described in Englyst et al. ${ }^{3}$

\section{Protein and lipid determination}

The crude protein of sorghum and wheat muffins was determined with the micro Kjeldahl method $^{\mathbf{1 6}}$ using a Kjeltec ${ } 2300$ Analyzer (Foss Tecator, Hoganas, Sweden). Muffins were ground and a $0.5 \mathrm{~g}$ sample was completely digested in $5 \mathrm{~mL}$ concentrated sulfuric acid with a catalyst tablet. The distillation and titration were automatically conducted by the Kjeldahl system. The crude fat in muffins was determined with a Soxtec Avanti 2055 system (Foss North America, MN, U.S.). ${ }^{17}$ The ground muffin samples were weighed into porous thimbles and extracted in ether, and the lipid was collected in extraction cups. After the evaporation of solvent and the drying process, the lipid was weighed and the crude fat percentage was calculated.

\section{Plasma glucose and insulin analysis}

Blood samples were collected at 15 minutes before and 0, 15, 30, 45, 60, 75, 90, 120, and 180 minutes after ingestion. Approximately $0.4 \mathrm{~mL}$ blood samples were collected with capillary tubes into a centrifuge tube and centrifuged at $5500 \times g$ with Microfuge ${ }^{\circledR}$ 22R Centrifuge (Beckman Coulter, Inc., Brea, CA, U.S.). Plasma was transferred and stored at $-20{ }^{\circ} \mathrm{C}$. Plasma glucose concentrations were measured with an ACE AleraTM Clinical Analyzer (West Caldwell, NJ, U.S.). Plasma insulin concentrations were measured using an insulin ELISA kit from Mercodia (Uppsala, Sweden).

\section{Statistical analysis}

All statistical analyses were conducted with the Statistical Analysis System (SAS, Release 9.2, SAS Institute Inc, Cary, NC). Values were expressed as means \pm standard error of the mean (SEM). The significance of differences among mean values was determined by analysis of variance (ANOVA). Differences were considered significant at $P<0.05$.

\section{Results and discussion}

\section{Flour composition and starch analysis}

For in vitro analysis, grain sorghum flour contained $22.7 \% \pm$ $0.8,43.8 \% \pm 0.8$, and $33.5 \% \pm 0.1$ for RDS, SDS, and RS, respectively. In comparison, wheat flour contained $37.5 \% \pm 0.3$, $47.4 \% \pm 0.4$, and $15.1 \% \pm 0.1$ for RDS, SDS, and RS measurement (Table 2). Raw grain sorghum flour exhibited significantly lower RDS content and significantly higher SDS and RS contents in comparison to raw wheat flour $(P<0.05)$. In a study investigating starch digestibility of cereals, researchers reported that unprocessed sorghum contained the highest amount of RS $(2-3 \times)$ and the lowest amount of RDS $\left(\mathrm{g} \mathrm{kg}^{-1} \mathrm{DM}\right)$ compared with wheat, barley, oat, maize, and rice. ${ }^{18}$ The present results also demonstrate a similar starch results with grain sorghum exhibiting $2 \times$ amount of RS and almost half the amount of RDS. The TS content for the two raw material flours measured $74.9 \%$
Table 2 In vitro total starch and starch fraction measurement of wheat and sorghum flours and muffins ${ }^{a}$

\begin{tabular}{llllll}
\hline & \multicolumn{2}{l}{ Wheat } & & \multicolumn{2}{l}{ Sorghum } \\
\cline { 2 - 3 } \cline { 5 - 6 } $\begin{array}{l}\text { Starch } \\
\text { composition }\end{array}$ & \multicolumn{1}{l}{ Flour } & Muffin & & Flour & Muffin \\
\hline TS\% & $64.5 \pm 0.5$ & $43.8 \pm 0.3$ & & $74.9 \pm 0.7$ & $49.2 \pm 0.8$ \\
RDS\% & $37.5 \pm 0.2$ & $88.2 \pm 0.3$ & & $22.7 \pm 0.8^{*}$ & $78.9 \pm 0.5 \dagger$ \\
SDS\% & $47.4 \pm 0.3$ & $11.5 \pm 0.1$ & & $43.8 \pm 0.8^{*}$ & $17.5 \pm 0.3 \dagger$ \\
RS\% & $15.1 \pm 0.1$ & $0.5 \pm 0.1$ & & $33.5 \pm 0.1^{*}$ & $3.6 \pm 0.3 \dagger$
\end{tabular}

${ }^{a}$ TS is represented on dry-basis; RDS, SDS, and SDS percentages are based on TS. Values reflect means \pm standard deviation; ${ }^{*}, \uparrow$ indicates $p<0.05$ significance.

for grain sorghum flour and $64.5 \%$ for wheat flour. Nearly identical grain sorghum TS content was also described in a study conducted by Austin et al. ${ }^{19}$ for Texas white sorghum of $74 \%$ TS and Sudanese grain sorghum flour from Abelgadir et al. ${ }^{11}$ In the present study, amylose contents of the initial flour material were measured to be $30.9 \% \pm 1.5$ for whole grain sorghum flour and $21.8 \% \pm 1.8$ for whole wheat flour. Dicko et al. ${ }^{5}$ reported the sorghum grain containing 60-75 $\mathrm{g}$ starch per $100 \mathrm{~g}$ fresh matter, consistent with current study data, and the amylose content of 12-22 $\mathrm{g}$ per $100 \mathrm{~g}$ of fresh matter. Gaffa et al. ${ }^{21}$ reported two Nigerian sorghum cultivars containing 25.5 and $25.7 \%$ amylose content and Sang et al. ${ }^{10}$ reported $24 \%$ amylose content in normal grain sorghum.

\section{Muffin composition and starch analysis}

The moisture content varied slightly, $35.2 \% \pm 0.3$ for sorghum muffins and $39.5 \% \pm 0.3$ for wheat muffins. Differences in batter water amounts correspond to moisture sample variance as explained in Materials and methods. Mean crude fat $( \pm \mathrm{SD})$ contents of sorghum and wheat muffins measured 18.5\% \pm 0.6 and $16.0 \% \pm 0.8$, respectively. Mean crude protein contents were $5.2 \% \pm 0.2$ for sorghum muffin and $7.8 \% \pm 0.3$ for wheat muffin, and ash contents were nearly identical for both samples with $2.2 \% \pm 0.1$ for sorghum and $2.0 \% \pm 0.1$ for wheat. Muffin ingredients were added identically between treatments, as listed in Table 1, except for flour and water amounts. Since more wheat flour was needed to provide $50 \mathrm{~g}$ of starch for each treatment, differences in protein content can be explained because of the different flour amount used owing to total starch content of wheat and sorghum flour. As Table 1 indicates, approximately $10.7 \mathrm{~g}$ more flour was required for wheat muffin treatments. Nutrition label information from material package stated both flour sources as containing identical amounts of protein, fat, sugar, and fiber. Differences in the crude fat and protein contents for final products can be explained by small differences of initial flour (not shown) when compositional analysis was carried out and results given on a dry-weight basis.

In the whole grain sorghum muffin, RDS contents were lower and SDS and RS contents were significantly higher compared with the control whole wheat muffin $(P<0.05)$ (Table 2$)$. The starch structure, particularly protein-starch interactions, may explain differences of starch digestion rates for treatments 
based on past research ${ }^{\mathbf{1 5 , 1 9 - 2 3}}$ or perhaps amylose content. In a study conducted by Sang et al., ${ }^{\mathbf{1 0}}$ raw isolated grain sorghum starches were investigated for in vitro starch digestibility with varying amylose contents, and authors found that the intermediate $(14.0 \%)$ amylose content of heterowaxy starch exhibited higher RS content rather than the amylose content of normal $(23.7 \%)$ starch. The heterowaxy starch with an intermediate amylose content exhibited slightly lower SDS yet the highest RS content, suggesting that amylose content is not necessarily responsible for predicting in vitro digestibility. Researchers also reported similar digestibility results for RDS and RS contents compared to grain sorghum flour used in the present study. The RDS content $(21.5 \%)$ of waxy grain sorghum starch and RS content (23.7\%) of heterowaxy grain sorghum starch are comparable to present materials analysis. ${ }^{\mathbf{1 0}}$ In contrast, normal, heterowaxy, and waxy raw sorghum starch samples exhibited higher amounts of SDS content (61.7-68.5\%), and lower amounts of amylose $23.7,14$, and $0 \%$, respectively. Findings from Benmoussa et al. ${ }^{24}$ also confirm variations in starch digestibility for three sorghum cultivars, suggesting that amylose content does not necessarily indicate the digestibility rate. Authors investigated different sorghum genotypes with ranging amylose content (19.2-22.4\%) and found that sample starch digestibility was mostly influenced by starch granule characteristics such as channel density which allows enzymes to attack the granule and the sorghum protein matrix, depending upon the sorghum genotype, also influences enzyme accessibility to granule. ${ }^{24}$ The protein content of the final product given to participants in the present study was relatively low; however, research has highlighted the protein-starch relationship in grain sorghum and its influence on starch digestion. In an article by Duodu et al. ${ }^{25}$ it has been discussed that exogenous factors such as the grain organizational structure, polyphenols, phytic acid, cell wall components, and starch are primary factors that influence protein digestibility, and additional researchers also confirmed ${ }^{26-29}$ results which explain the coordinated relationship between sorghum starch and protein digestibility. As researchers have discovered that both starch granules and protein bodies are closely associated with each other; enzymatic accessibility is greatly hindered for starch gelatinization and digestibility, which may contribute to poor protein quality of sorghum. ${ }^{25}$ In a more recent publication from Mokrane et al. ${ }^{26}$ regarding the protein, Algerian sorghum cultivars were only evaluated for protein digestibility. Researchers found that the protein content in cultivars could provide good quantity and quality of essential amino acids though the digestibility varied greatly between cultivars and unfortunately starch digestion was not analyzed. The Algerian sorghum flours contained $12.6-16.4 \%$ crude protein (DB), approximately $4-8 \%$ higher than grain sorghum flour used in the present study.

\section{Incremental glucose and insulin results}

Fig. 1 and 2 show incremental changes of plasma glucose and plasma insulin concentrations over experiment duration. With the grain sorghum muffin treatment, the glucose responses

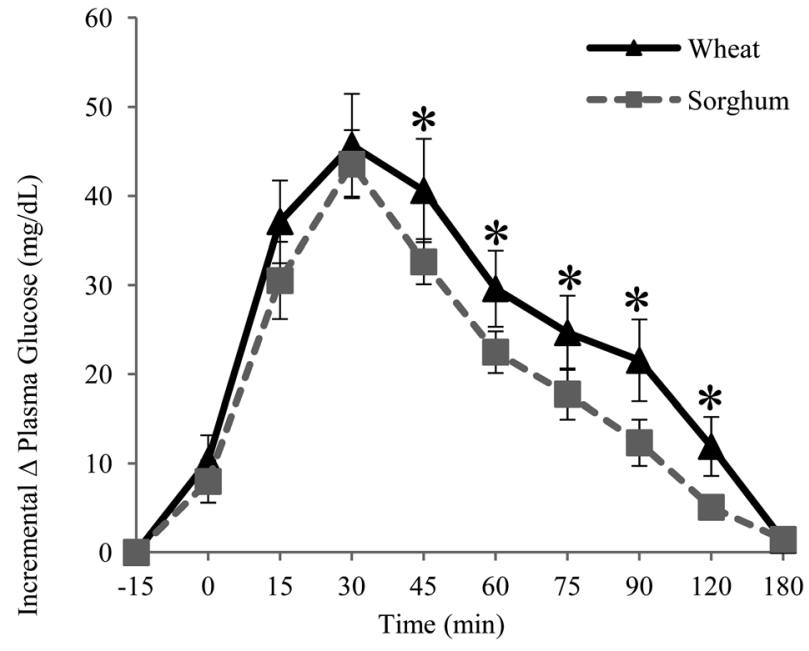

Fig. 1 Mean incremental change of plasma glucose in healthy men ( $n$ $=10$ ) with standard error of mean bars (SEM). * $p<0.05$ at time intervals.

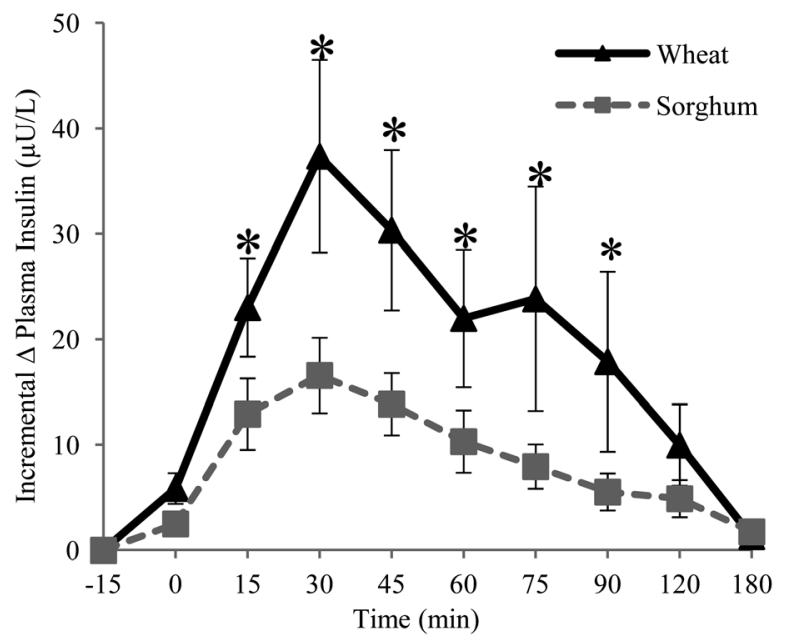

Fig. 2 Mean incremental change of plasma insulin in healthy men ( $n=$ 10) with standard error of mean bars (SEM). ${ }^{*} p<0.05$ at time intervals.

from participants were significantly lower at 45, 60, 75, 90, and 120 minute intervals $(P<0.05)$.

Mean iAUC responses of glucose were significantly reduced about $26 \%$ from $3863 \pm 443$ to $2871 \pm 163 \mathrm{mg}(\sim 3 \mathrm{~h}) \mathrm{dL}^{-1}$ as shown in Fig. $3(P<0.05)$. Incremental insulin concentrations for the sorghum muffin treatment were significantly lower at 15 , $30,45,60,75$, and 90 minutes $(P<0.05)$ and were also significantly reduced about $55 \%$ from $3029 \pm 965$ to $1357 \pm 204 \mu \mathrm{U}$ ( 3 h) L $\mathrm{L}^{-1}$ (Fig. 3).

Processing and cooking conditions do influence starch hydrolysis. ${ }^{\mathbf{1 2 , 3 0}}$ In one human study conducted by Lakshmi and Vimala, ${ }^{12}$ the study observed glucose responses from six noninsulin dependent diabetic patients, and researchers compared the three sorghum recipes, both with whole grain and dehulled grains, and also compared to either wheat or rice recipe controls, additionally. Recipes were traditional dietary foods in 


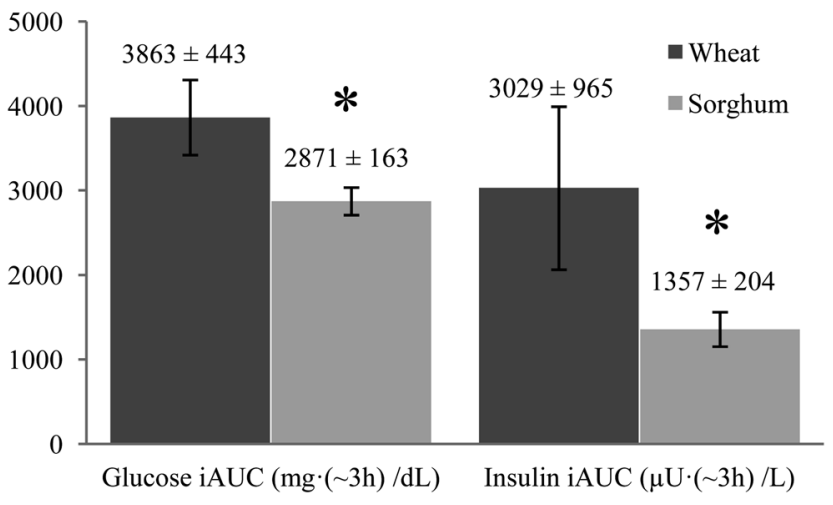

Fig. 3 Total mean incremental area under the curves (iAUC) for plasma glucose and insulin responses of healthy males $(n=10)$ with standard error of mean (SEM) values. ${ }^{*} p<0.05$ significance.

different preparation forms including a shallow pan-fried product, boiled, and a fermented-steamed product. Researchers used larger treatment samples (85-90 g carbohydrate), but with all three samples, and whole sorghum food recipes exhibited lowest plasma glucose and insulin responses compared to identical dehulled sorghum recipes and controls. In addition, whole grain sorghum foods showed an average of two-fold amount of fiber content compared to dehulled sorghum foods. Researchers investigating Sudanese cereal foods and their glucose and insulin responses have reported wheat and millet as having slightly lower glucose and insulin incremental areas under the curve compared to sorghum,${ }^{\mathbf{1 1}}$ however, flours used for the study did not provide total starch content for various treatment grains and forms to confirm identical treatment conditions. With the literature available, it is evident that processing conditions such as de-hulling may greatly influence overall glucose and insulin responses as reported by Taylor and Emmambux, ${ }^{6}$ so it is important for human studies to test whole grain sorghum for potential functional food ingredients. Cultivars bred for functional starch properties would be excellent candidates for functional food ingredients, however, the preparation, processing, and cooking conditions of the final product remain central to evaluate effectiveness.

\section{Conclusions}

Results show whole grain sorghum as a good functional food ingredient for controlling glucose and insulin levels in healthy humans. Compared to wheat, functional starch fractions for sorghum showed increased SDS and RS contents and lower RDS content and decreased glucose and insulin responses in healthy humans. Research implies further investigation with pre-diabetic or diabetic individuals would be beneficial for providing more additional analysis of grain sorghum as a functional ingredient. Additionally, future research may include sensory evaluation for the ingredient profile and functionality. With increasing gluten-intolerance and environmental concerns, grain sorghum will continue to be utilized for future research applications.

\section{Acknowledgements}

Support for this research from the Arkansas Grain Sorghum Promotion Board is gratefully appreciated.

\section{References}

1 Centers for Disease Control and Prevention, National diabetes fact sheet: national estimates and general information on diabetes and prediabetes in the United States, U.S. Department of Health and Human Services, Centers for Disease Control and Prevention, Atlanta, GA, 2011.

2 G. Annison and D. L. Topping, Annu. Rev. Nutr., 1994, 14, 297-320.

3 H. N. Englyst, S. M. Kingman and J. H. Cummings, Eur. J. Clin. Nutr., 1992, 46(2), s33-s50.

4 Food and Agriculture Organization (FAO), FAO Statistics of Sorghum Crop Production, 2010.

5 M. H. Dicko, H. Gruppen, A. S. Traoré, A. G. J. Voragen and W. J. H. van Berkel, Afr. J. Biotechnol., 2006, 5(5), 384395.

6 J. R. N. Taylor and M. N. Emmambux, Molecular Diversity and Health Benefits of Carbohydrates from Cereals and Pulses, 2010, 87(4), 263-271.

7 M. J. Gidley, P. A. Pluschke, P. A. Sopade, G. J. S. Al-Rabadi, A. Sultan, C. Y. Gan, X. Li, D. Zhang and W. L. Bryden, Australian Poultry Science Symposium, 2011, 22, 139146.

8 R. N. Taylor, T. J. Schober and S. R. Bean, J. Cereal Sci., 2006, 44, 252-271.

9 L. W. Rooney and R. L. Pflugfelder, J. Anim. Sci., 1986, 63, 1607-1623.

10 Y. Sang, S. Bean, P. A. Seib, J. Pedersen and Y. C. Shi, J. Agric. Food Chem., 2008, 56, 6680-6685.

11 M. Abdelgadir, M. Abbas, A. Jarvi, M. Elbagir, M. Elton and C. Berne, Diabetic Med., 2005, 22, 213-217.

12 K. B. Lakshmi and V. Vimala, J. Nutr., 1996, 16, 1651-1658.

13 A. C. Carciofi, F. S. Takakura, L. D. De-Oliverira, E. Teshima, J. T. Jeremais, M. A. Brunetto and F. Prada, J. Anim. Physiol. Anim. Nutr., 2008, 92, 326-336.

14 L. D. De-Oliveira, A. C. Carciofi, M. C. C. Oliveira, R. S. Vascocellos, R. S. Bazolli, G. T. Pereira and F. Prada, J. Anim. Sci., 2008, 86, 2237-2246.

15 J. H. Wong, T. Lau, N. Cai, J. Singh, J. F. Pedersen, W. H. Vensel, W. J. Hurkman, J. D. Wilson, P. G. Lemaux and B. B. Buchanan, J. Cereal Sci., 2009, 49, 73-82.

16 American Association of Cereal Chemists (AACC) international, Approved Methods of Analysis, 10th edn. Method 46-13. Crude Protein - Micro-Kjeldahl Method. Method 30-10. Crude Fat in Flour, Bread, and Baked Cereal Products Not Containing Fruit.

17 A. M. AwadElkareem and J. R. N. Taylor, Cereal Chem., 2012, 88(4), 334-348.

18 G. Giuberti, A. Gallo, C. Cerioli and F. Masoero, Anim. Feed Sci. Technol., 2012, 174, 163-173.

19 D. L. Austin, N. D. Turner, C. M. McDonough and L. W. Rooney, Cereal Chem., 2012, 89(4), 190-197. 
20 S. Serna-Saldivar and L. W. Rooney, Sorghum and Millets: Chemistry and Technology, ed. D. A. V. Dendy, AACC International, St. Paul, MN, 1995, pp. 69-124.

21 T. Gaffa, Y. Yoshimoto, I. Hanashiro, O. Honda, S. Kawasaki and Y. Takeda, Cereal Chem., 2004, 80(2), 255-260.

22 G. Zhang and B. R. Hamaker, Cereal Chem., 1998, 75, 710713.

23 U. Lehmann and F. Robin, Trends Food Sci. Technol., 2007, 18(7), 346-355.

24 M. Benmoussa, B. Suhendra, A. Aboubacar and B. R. Hamaker, Starch/Staerke, 2006, 58(2), 92-99.
25 K. G. Duodu, J. R. N. Taylor, P. S. Belton and B. R. Hamaker, J. Cereal Sci., 2003, 38(2), 117-132.

26 H. Mokrane, H. Amoura, N. Belhaneche-Bensemra, C. M. Courtin, J. A. Delcour and B. Nadjemi, Food Chem., 2010, 121(3), 719-723.

27 L. I. Ezeogu, K. G. Duodu and J. R. N. Taylor, J. Cereal Sci., 2005, 42(1), 33-44.

28 P. Rooney, J. Agric. Food Chem., 1986, 63, 1607-1623.

29 G. Zhang and B. R. Hamaker, Cereal Chem., 2005, 82, 534540.

30 N. R. Dlamini, J. R. N. Taylor and L. W. Rooney, Food Chem., 2007, 105, 1412-1419. 